\title{
Complete Androgen Insensitivity Syndrome (Testicular Feminization Syndrome): An Interesting Case of Primary Amenorrhoea with 46XY Karyotype
}

\author{
Shreya Hegde ${ }^{1}$, Vinitha Samartha ${ }^{2}$, Thoppil Reba Philipose ${ }^{3}$ \\ ${ }^{1,2,3}$ DepartmentOf Pathology, AJ Institute of Medical Sciences And Research Centre, Karnataka, India
}

\begin{abstract}
Androgen insensitivity (testicular feminization) syndrome is a rare inherited form of male pseudohermaphroditism that occurs in a phenotypically normal woman with male karyotype (XY). We present a case of a 20 years old patient who had history of primary amenorrhea. The clinical examination shows a female phenotype: the breasts are normally developed, absence of hair in the groins and axillary areas, short blind vagina, absent cervix and uterus. A firm mass measuring $5 \times 3 \mathrm{cms}$ was palpable in the left inguinal region. MRI pelvis revealed a cystic lesion measuring $6.8 \times 5.5 \mathrm{cms}$ on the right side. Histopathological examination revealed that both the masses were composed of testicular tissue with absence of ovarian component. The testes were removed to avoid the risk of malignancy. The patient was counseled and discharged with lifelong hormone replacement therapy.
\end{abstract}

Keywords:testicular feminization syndrome, primary amenorrhea, androgen insensitivity syndrome, SRY gene

\section{Introduction}

Androgen insensitivity (testicular feminization) syndrome is a rare inherited form of male pseudohermaphroditism that occurs in a phenotypically normal woman with male karyotype (XY). ${ }^{1}$ The first medical report on Androgen insensitivity syndrome (AIS) was published in 1953 by J.M. Morris, an American gynaecologist. The syndrome was designated "testicular feminization syndrome" because testes produces hormones with oestrogen like actions. ${ }^{2}$

AIS results from an androgen receptor defect. The probable explanation of the syndrome is the absence of the cytosol androgen binding protein receptor that is normally present in the androgen responsive tissues. So the male fetus is not stimulated by normal levels of circulating androgens. As a result, there is no fusion of the genital folds to form the scrotum and penis and no posterior migration of the labioscrotal folds. ${ }^{1}$

The estimated prevalence of AIS is between 1 in 20,000 and 1 in 99,000 genetic males. AIS is thought to be related to approximately $6-10 \%$ of cases of primary amenorrhea. ${ }^{3}$

The aim of this paper is to present the importance and diagnosis of a case of CAIS and discussions that follow it.

\section{Case Report}

A 20 year old woman was admitted for investigations of primary amenorrhea. Physical examination revealed a female phenotype with normal development of breasts and normal external genitalia but with absence of hair in the groins and axillary area (Figure 1). Systemic examination revealed a firm mass measuring $5 \times 3 \mathrm{cms}$ in the left inguinal region. Gynecological examination revealed a short blind vagina $(3 \mathrm{cms}$ in length) with absence of uterus and cervix.

Magnetic resonance imaging showed a well defined lesion measuring $5.4 \times 2.3 \mathrm{cms}$ in left inguinal region suggestive of testicular parenchyma and ovarian follicles. A cystic lesion measuring 6.8 x $5.5 \mathrm{cms}$ with a well-defined solid altered signal area adjacent to it measuring $3.3 \times 3 \mathrm{cms}$ was seen in the pelvis on right side. Hormonal assay showed FSH levels of $13.9 \mathrm{mIU} / \mathrm{ml}$, elevated LH levels of $29.8 \mathrm{mIU} / \mathrm{ml}$ and serum testosterone levels of $551.99 \mathrm{ng} / \mathrm{dL}$. Tumour markers revealed serum $\beta \mathrm{HCG}-<1.2 \mathrm{mIU} / \mathrm{mL}$, serum AFP $-0.44 \mathrm{IU} / \mathrm{mL}$ and LDH - 436 IU/L. A karyotype was also performed which revealed a 46 XY complement.

In view of these findings, a diagnosis of Complete androgen insensitivity syndrome was made. The patient was counselled regarding the need for gonadectomy in view of increased risk of malignancy and also regarding the need for lifelong hormone replacement therapy. Bilateral gonadectomy was done by laparoscopy and the specimen sent for histopathological examination (Figure 2).

Gross examination of the left gonadectomy specimen revealed a smooth, glistening mass with a solid grey-brown cut surface (Figure 3). Microscopy showed seminiferous tubules lined predominantly by Sertoli cells with peritubular fibrosis (Figure 4). Germ cells were reduced in number with maturation arrest at primary and secondary spermatocyte stage. The lumen did not show mature spermatozoa. The interstitium showed Leydig cell hyperplasia. 
Gross examination of the right gonadectomy specimen revealed a smooth, glistening mass with a solid grey-brown cut surface along with a unilocular cyst measuring 5x2 cms (Figure 3). Microscopy showed solid and cystic components. The solid component consisted of immature testicular tissue with maturation arrest of spermatogonia. The interstitium showed Leydig cell hyperplasia. The cystic component showed a Wolffian cyst lined by cuboidal, predominantly non ciliated, epithelium resting on the well-developed basement membrane (Figure 5). No signs of testicular cancer were identified in both the gonads.

The patient was put on oestrogen therapy and informed that she could have a normal sexual life but would be infertile.

\section{Discussion}

Testicular Feminization, or Androgen Insensitivity Syndrome, is a rare disease when a male, genetically XY, because of various abnormalities of the X chromosome, has some physical characteristics of a woman, or even a full female phenotype. ${ }^{4}$

Based on genital phenotype AIS is traditionally classified into 3 clinical subgroups - Complete, Partial and Mild. Complete Androgen Insensitivity Syndrome (CAIS) individuals have normal female external genitalia and typically present either before puberty with masses in the inguinal canal that are subsequently identified as testes or at puberty with primary amenorrhea and sparse to absent pubic and/or axillary hair. Partial Androgen Insensitivity Syndrome (PAIS) individuals are predominantly female, predominantly male, or ambiguous genitalia with signs of external genital masculinization including clitoromegaly or posterior labial fusion. Mild Androgen Insensitivity Syndrome (MAIS) individuals have typical male genitalia and usually present with gynaecomastia at puberty while in some instances, the only observed abnormality appears to be male infertility. ${ }^{5}$

CAIS, also known as Morris syndrome, results from an absence or abnormal cytosol receptor for androgens. Thus, despite possessing a male karyotype (46XY), phenotypically patient presents as an asymptomatic female usually discovered during the perimenarchal stage when the individual fails to menstruate. The testis is often undescended and the ovaries, uterus, fallopian tubes, and upper third of vagina are typically absent. Externally, the labia majora and minora are usually well formed. ${ }^{6}$ The underlying cause is a mutation in the androgen receptor gene localized in the long arm of $\mathrm{X}$ chromosome Xq11-12.2. Although $70 \%$ of these mutations are $\mathrm{X}$-linked recessive, $30 \%$ can also be sporadic de novo mutations.

During the $6^{\text {th }}$ week of male foetal development, under the influence of the SRY gene located on the Y chromosome, the testes begin to differentiate from the genital ridges. Leydig cells appear through the end of the $8^{\text {th }}$ week, and they start producing testosterone. In the absence of testosterone, female sexual characteristics develop. ${ }^{8}$

Gonadectomy is currently recommended for AIS with cryptorchidism due to high risk of germ cell malignancy. The risk of malignancy of undescended intra-abdominal testes is $3.6 \%$ at 25 years and $33 \%$ at 50 years. ${ }^{9}$ Hormone replacement therapy is required after gonadectomy, and should be modulated over time to replicate the hormone level naturally present in the body during various stages after puberty. ${ }^{10}$

Thus, CAIS is a rare disease that must be diagnosed and treated through close work between gynaecologists, endocrinologists, pathologists and psychiatrists. ${ }^{4}$

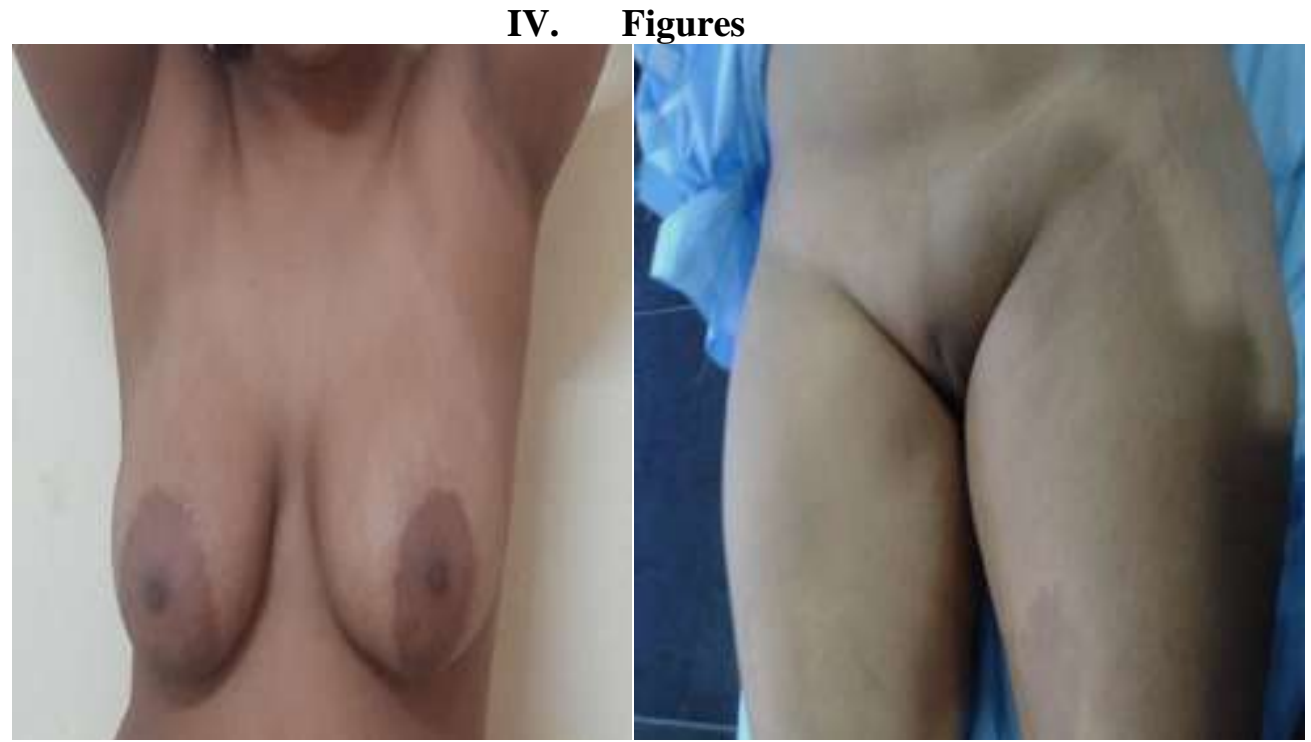

Fig1: Physical examination revealed normal development of breasts and normal external genitalia but with absence of hair in the groins and axillary area. 


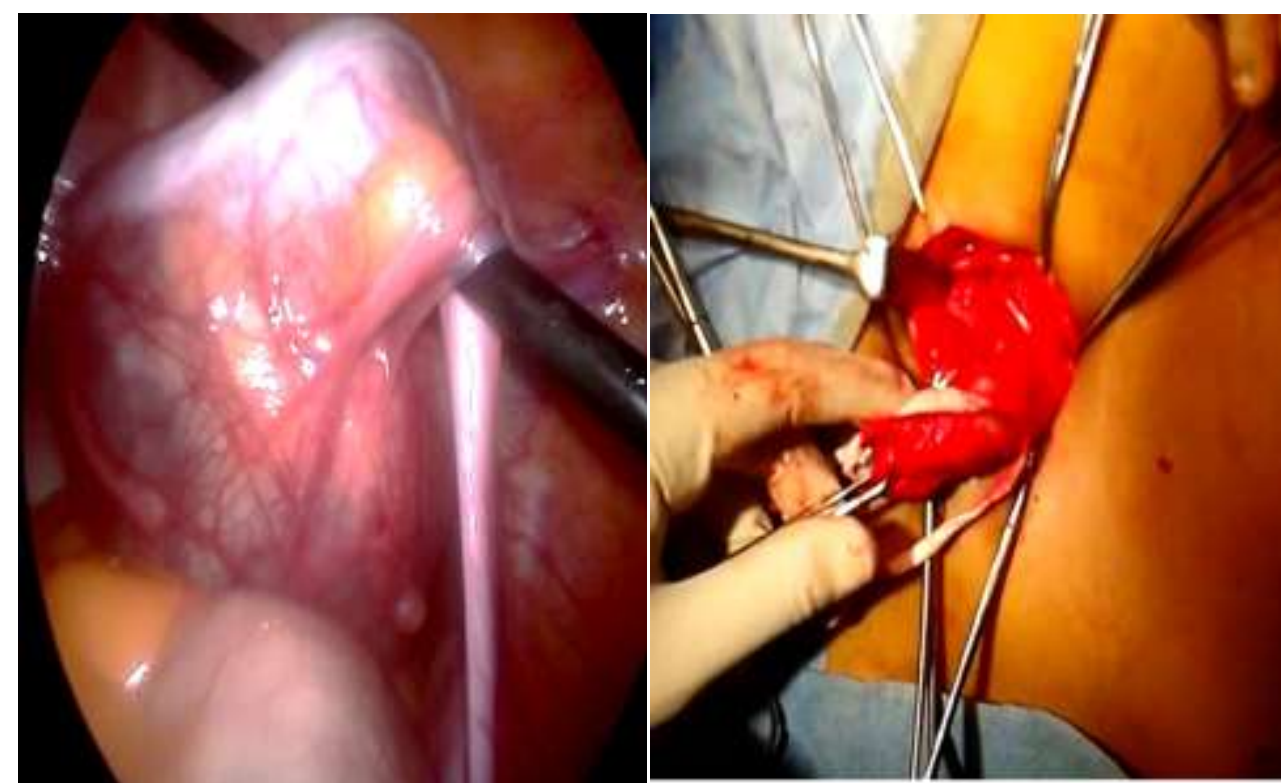

Fig2: Bilateral gonadectomy done by laparascopy.

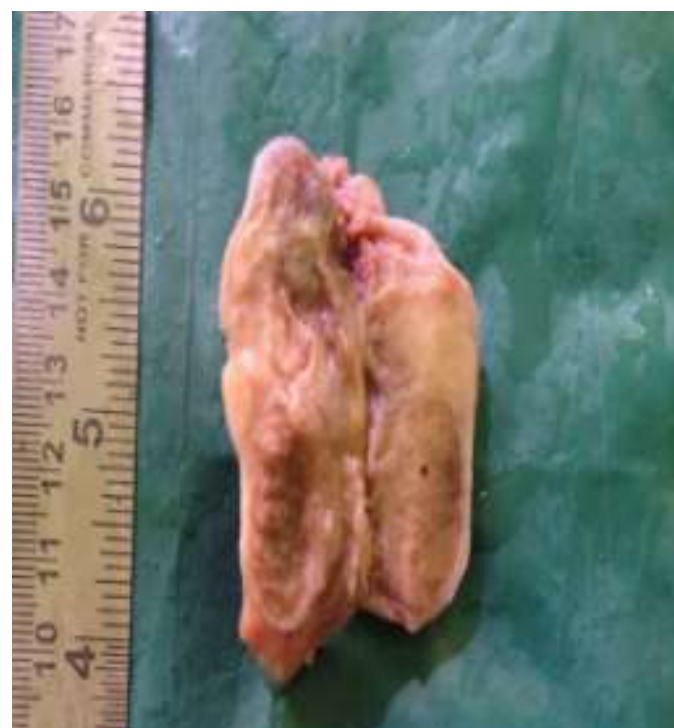

(HPE 407/15)

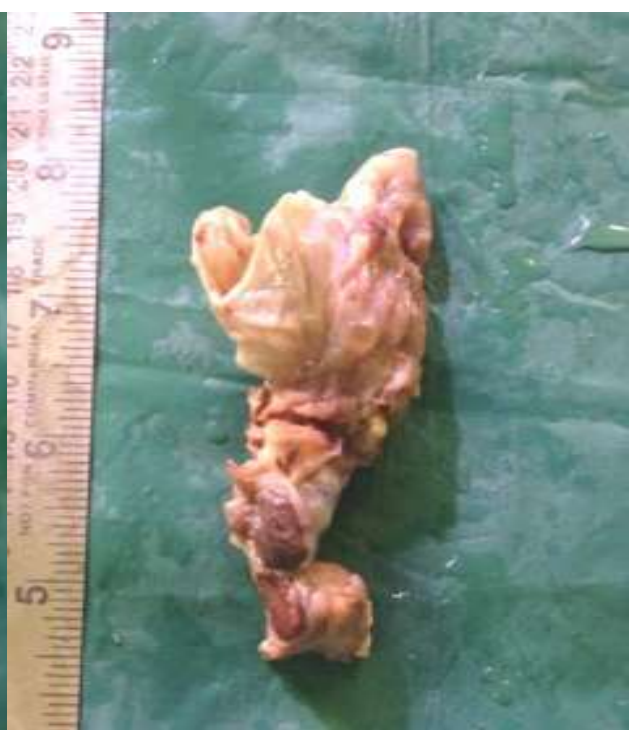

(HPE 397/15)

Fig. 3: Gross examination of Left gonadectomy (HPE 407/15) and Right gonadectomy (HPE 397/15) specimen revealed a smooth, glistening mass with a solid grey-brown cut surface. The right gonadectomy specimen also revealed a unilocular cyst measuring $5 \times 2 \mathrm{cms}$.

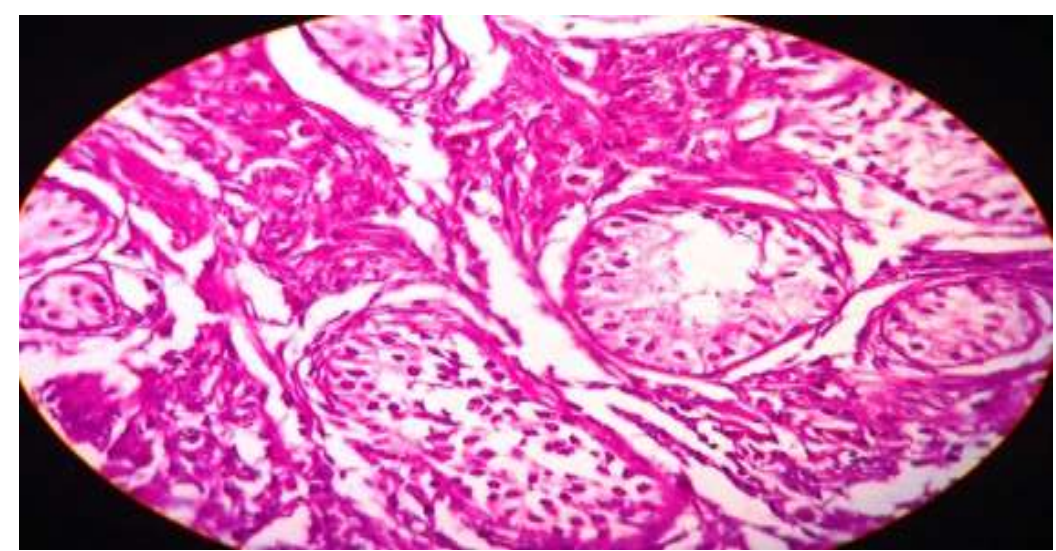

Fig. 4: Microscopy of left gonadectomy specimen showing seminiferous tubules with peritubular fibrosis. (H\&E stain,40x) 


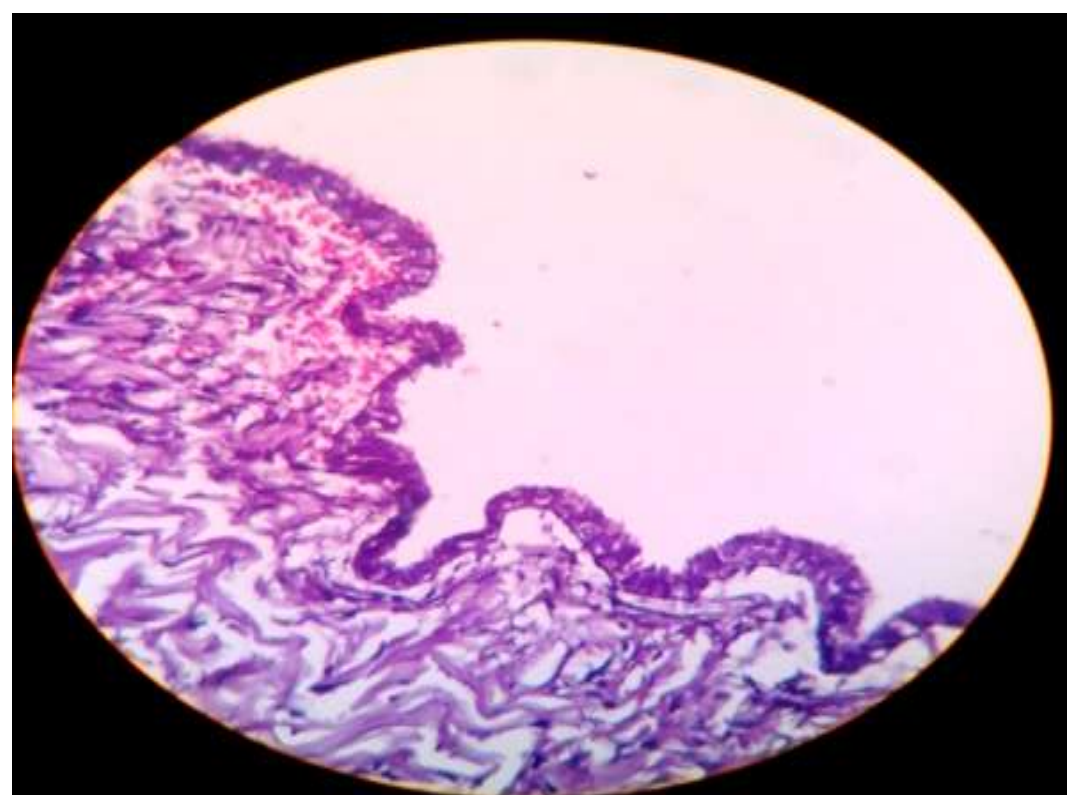

Fig. 5: Cystic component of right gonadectomy specimen showing a Wolffian cyst lined by cuboidal, predominantly non ciliated epithelium. (H\&E stain,40x)

\section{Acknowledgement}

The authors have no conflict of interest to declare. This study was not supported by any funding agency.

\section{References}

[1]. Zorlu F, Cengiz M, Gurkaynak M, Yildiz F, Atahan LI. Seminoma arising in androgen insensitivity syndrome (testicular feminization syndrome) : A case report. Turkish Journal Of Cancer 2001; 31(4):168-71.

[2]. Yuan X, De-fen W, Xiao-ying L, Jun Y, Wei W. Mutation of the androgen receptor in an adolescent with complete androgen insensitivity syndrome. Chinese Medical Journal 2010;123(1):1473-6.

[3]. Yamini P, Ajinkya J, Bharat K, Ushang K, Prashant K. A case of primary amenorrhea with 46XY karyotype: Androgen insensitivity syndrome. IOSR Journal of Pharmacy and Biological Sciences 2013;5(5): 36-9.

[4]. Constantin G, Alexandru D, Sorin P, et al. Testicular feminization : complete androgen insensitivity syndrome. Discussions based on a case report. Rom J MorpholEmbryol 2014;55(1):177-81.

[5]. Gottlieb B, Beitel L, Trifiro M. Androgen insensitivity syndrome, Gene reviews 1999. Available at :http://www.geneclinics.org/profiles/androgen/detail.html.

[6]. Rasalkar D, Paunipagar b, Ng A, Lai F, Bagaria S. Intra-abdominal testicular seminoma in a woman with testicular feminization syndrome. Case reports in radiology 2011; Article ID 592124.

[7]. Chauhan N, Sharma Y, Sharma S, Sharma S. Complete androgen insensitivity syndrome with microtia:A rare presentation, Japanese journal of radiology 2011; 29:67-71.

[8]. Hughes IA, Deeb A. Androgen resistence. Best Pract Res ClinEndocrinolMetab 2006; 20(4):577-98.

[9]. Dejager S, Bry-Gauillard H, Bruckert E, et al. A comprehensive endocrine description of Kennedy's disease revealing androgen insensitivity linked to CAG repeat length. J ClinEndocrinolMetab 2002; 87(8):3893-901.

[10]. Ahmed SF, Cheng A, Dovey L, et al. Phenotypic features, androgen receptor binding, and mutational analysis in 278 clinical cases reported as androgen insensitivity syndrome. J ClinEndocrinolMetab 2000; 85:658-65. 